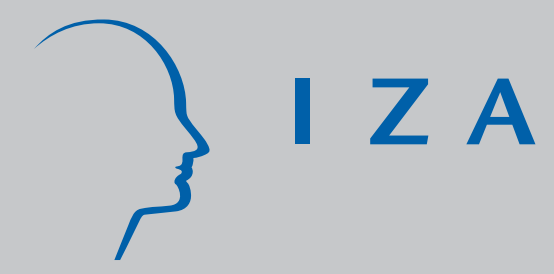

IZA DP No. 3697

Variable Search Intensity in an Economy with Coordination Unemployment

Leo Kaas

September 2008 


\title{
Variable Search Intensity in an Economy with Coordination Unemployment
}

\author{
Leo Kaas \\ University of Konstanz \\ and IZA
Discussion Paper No. 3697
September 2008 \\ IZA \\ P.O. Box 7240 \\ 53072 Bonn \\ Germany \\ Phone: +49-228-3894-0 \\ Fax: +49-228-3894-180 \\ E-mail: iza@iza.org
}

Any opinions expressed here are those of the author(s) and not those of IZA. Research published in this series may include views on policy, but the institute itself takes no institutional policy positions.

The Institute for the Study of Labor (IZA) in Bonn is a local and virtual international research center and a place of communication between science, politics and business. IZA is an independent nonprofit organization supported by Deutsche Post World Net. The center is associated with the University of Bonn and offers a stimulating research environment through its international network, workshops and conferences, data service, project support, research visits and doctoral program. IZA engages in (i) original and internationally competitive research in all fields of labor economics, (ii) development of policy concepts, and (iii) dissemination of research results and concepts to the interested public.

IZA Discussion Papers often represent preliminary work and are circulated to encourage discussion. Citation of such a paper should account for its provisional character. A revised version may be available directly from the author. 
IZA Discussion Paper No. 3697

September 2008

\section{ABSTRACT \\ Variable Search Intensity in an Economy with Coordination Unemployment ${ }^{*}$}

This paper analyzes an urn-ball matching model in which workers decide how intensively they sample job openings and apply at a stochastic number of suitable vacancies. Equilibrium is not constrained efficient; entry is excessive and search intensity can be too high or too low. Moreover, an inefficient discouraged-worker effect among homogenous workers emerges under adverse labor market conditions. Unlike existing coordination-friction economies with fixed search intensity, the model can account for the empirical relation between the job-finding rate and the vacancy-unemployment ratio, provided that search costs are small and that search intensity is sufficiently procyclical.

JEL Classification: E24, J63, J64

Keywords: matching function, coordination frictions, unemployment

Corresponding author:

Leo Kaas

Department of Economics

University of Konstanz

Box D145

78457 Konstanz

Germany

E-mail: Leo.Kaas@uni-konstanz.de

\footnotetext{
* I am grateful to Pieter Gautier and to audiences at the Penn S\&M workshop, the NBER Summer Institute 2008, and at the Universities of Bonn and Vienna for their comments. Support by the German Research Foundation is gratefully acknowledged.
} 


\section{Introduction}

Search and matching models are widely used to address various labor market phenomena, such as unemployment, worker and job flows, and wage dispersion. A large portion of the literature utilizes the idea of a reduced-form matching function which maps the stocks of searching workers and firms into the flow of new matches. Despite of its modeling advantages, this approach suffers from two limitations. One is its inability to deal with heterogeneity convincingly. Of course, the foremost purpose of the matching function is to abstract from any explicit source of frictions (including heterogeneity) to describe the implications of costly trading in the labor market with a minimum amount of complexity. Yet, many important issues (for example, the pattern of skill premia) require an explicit analysis of how heterogeneity affects labor market outcomes. ${ }^{1}$ The other limitation is that a reduced-form matching function is, by construction, invariant to policy. Again a more explicit model of frictions is needed to address how policy affects the matching relationship (see also Lagos (2000) and Shimer (2007)).

There is a large literature on microeconomic foundations behind the aggregate matching function; see section 3 of Petrongolo and Pissarides (2001) for a survey. One such foundation rests on coordination frictions; early contributions are Butters (1977), Hall (1977) and Montgomery (1991), more recent ones are Burdett, Shi, and Wright (2001), Julien, Kennes and King (2000, 2006) and Albrecht, Gautier, and Vroman (2006). The key idea is simple: since workers do not coordinate their application decisions and firms do not coordinate their job-offer decisions, some firms end up with no applications while others get many, and some workers obtain several job offers while others have none. So at the end of every period, unfilled jobs and unemployed workers coexist. These models give rise to aggregate matching functions which typically have constant returns in economies with a large number of workers and jobs.

Still there are a few open issues with coordination-friction models. One is that search intensity is typically held constant. Although it is straightforward to include variable

\footnotetext{
${ }^{1}$ There is a number of papers utilizing reduced-form matching functions in models with heterogenous jobs or heterogenous skills (e.g. Acemoglu (2001) and Albrecht and Vroman (2002)). But such models must rest on ad-hoc assumptions on how workers and jobs of different types are matched.
} 
search intensity in standard search models with exogenous matching functions (see Chapter 5 of Pissarides (2000)), it is a less obvious matter in economies where matching frictions result explicitly from coordination problems. Work by Albrecht, Gautier, and Vroman (2006), Galenianos and Kircher (2008) and Kircher (2007) identifies search intensity with the number of applications that a worker sends. However, because of the discrete-choice nature of the worker's decision problems, equilibrium is difficult to characterize analytically. Also it is not obvious whether the number of applications is the right measure for search intensity. Chance plays an important role in the search for jobs; some workers who search hard may simply be unlucky, find few suitable job openings and send few applications. Others who spend less time on search, may notice a larger number of adequate job openings and send more applications. A second open issue is quantitative: can coordination-friction models match the empirical relation between the vacancy-unemployment ratio and the job-finding rate? Recently, Mortensen (2007) and Shimer (2007) have analyzed microfoundations of the matching function which are based on mismatch and which generate a reasonable fit of the matching function and of the Beveridge curve. To my knowledge, these quantitative features have not been explored for models with coordination frictions thus far. ${ }^{2}$

This paper addresses these two issues. The first contribution is theoretical and is the content of Section 2. I analyze an urn-ball matching model in which workers decide about the rate at which they sample job openings ("search intensity") and apply at all suitable jobs they observe. For a given search intensity, the actual number of suitable jobs (and so the number of applications) is stochastic. The expected number of applications, however, increases proportionately with search intensity. As applications are sent randomly, wages are determined by ex-post competition, according to the same bidding game as in Julien, Kennes and King (2000, 2006) and Albrecht, Gautier, and Vroman (2006). Workers with at least two offers receive the competitive wage, those who have only one offer are paid the reservation wage. A key advantage of my model is that search intensity is a continuous choice variable, which makes the model tractable and allows for an explicit equilibrium characterization using first-order conditions. When labor market conditions are good, there are many job openings per worker and all workers search with the same intensity. With less

\footnotetext{
${ }^{2}$ Julien, Kennes, and King (2006) examine a coordination-friction model quantitatively, but their focus is wage dispersion, and not the matching-function elasticity.
} 
favorable conditions, however, there are fewer job openings and it may happen that no symmetric equilibrium in pure search-intensity strategies exists. Instead, some workers are active and search with a common positive intensity, while others remain inactive and decide not to search at all. Thus, the model can describe endogenous nonparticipation in an environment where all workers are equally productive and have the same taste for leisure. When comparing these equilibrium outcomes to the choice of a social planner, I obtain the following results: (i) nonparticipation is never constrained efficient; (ii) entry is always excessive, for the same reason as in Albrecht, Gautier, and Vroman (2006), and (iii) search intensity can be too high or too low.

Section 3 contains the quantitative exploration of this model. In Section 3.1 I show that existing coordination-friction models, with fixed search intensity and with a reasonable choice of the period length, are unable to account for the empirical elasticity of the matching function. Specifically, the job-finding rate responds too little to variations in the vacancy-unemployment ratio. I then go on to examine a dynamic version of my model in which the parameters of a reduced-form search cost function are calibrated so as to match both the mean job-finding rate and its elasticity with respect to the vacancy-unemployment ratio. I find that search costs are quantitatively small (less than $1 \%$ of the utility flow of an unemployed worker) but search intensity responds strongly and positively to productivity. Nonetheless, variable search intensity amplifies the economy's reaction to a productivity shock only little.

\section{The static model}

\section{$2.1 \quad$ The setup}

Consider a one-period economy with a large number $M$ of identical workers and a large number of $N$ of identical firms, each creating one vacancy. The number of workers is fixed, but the number of firms is determined from a free-entry condition. I consider the limit where both $M$ and $N$ tend to infinity and where $q=M / N$, the number of workers per job opening, is positive and finite. All agents are risk neutral and aim to maximize their expected income net of search costs. At the end of the period, unemployment income is zero and employed workers produce $p$ units 
of output (=job surplus). I consider the following sequence of events.

Stage I Firms enter at marginal cost $c(1 / q)$ where $c$ is a weakly increasing function of the number of active firms per worker.

Stage II Every worker decides search intensity $\lambda$ at cost $k(\lambda)$, where $k$ is increasing and convex in $\lambda \geq 0$. If a worker searches with intensity $\lambda$, he observes a suitable vacancy at any given firm with probability $\lambda / N$ and applies there. These stochastic events are independent across workers and firms.

Stage III Each firm makes a wage offer to at most one applicant, rejecting all others.

Stage IV Workers credibly reveal to firms how many offers they have, and firms can simultaneously revise their initial bids.

Stage V Workers decide what offer (if any) to accept.

I impose the usual anonymity restriction that every worker treats all (identical) firms equally (at stage $\mathrm{V}$ ) and that every firm treats all workers equally (at stage III).

Two remarks are in order. First, the specification that marginal entry costs are not constant is needed to limit entry in an equilibrium where some workers are inactive. The assumption can be justified, for example, by non-labor inputs in fixed supply (e.g. land) whose prices increase in the number of active firms. Second, search intensity is a continuous variable which determines the likelihood $\lambda / N$ that a worker observes a suitable job opening at any firm. This likelihood is plausibly proportional to $1 / N$ : the worker samples a certain (random) segment of the labor market whose size increases with search intensity $\lambda$. If the number of firms becomes larger, the size of the sampled segment stays the same, but the probability that a given firm belongs to this segment falls with factor $1 / N$.

In the large economy, the number of applications (per worker and per job) are Poisson distributed. A worker with search intensity $\lambda$ applies at exactly $n$ firms with probability

$$
\left(\begin{array}{l}
N \\
n
\end{array}\right)\left(\frac{\lambda}{N}\right)^{n}\left(\frac{N-\lambda}{N}\right)^{N-n} \approx \frac{1}{n !} \lambda^{n} e^{-\lambda} .
$$


Conversely, if all workers search with intensity $\lambda$, a firm receives applications from exactly $m$ workers with probability

$$
\left(\begin{array}{c}
M \\
m
\end{array}\right)\left(\frac{\lambda}{N}\right)^{m}\left(\frac{N-\lambda}{N}\right)^{M-m} \approx \frac{1}{m !}(\lambda q)^{m} e^{-\lambda q}
$$

Thus the expected number of applications per worker is $\lambda$ and the expected number of applications per firm is $\lambda q$.

The last three stages of this game have the following solution. Firms offer the reservation wage at stage III, revising the offer at stage IV only if the worker reveals another offer, in which case Bertrand competition drives wage offers to the marginal product. At the last stage, anonymity implies that workers randomize between equal offers. In this respect, my model resembles those of Julien, Kennes, and King (2000) and Albrecht, Gautier, and Vroman (2006) where workers with only one offer are paid the monopsony wage and workers with multiple offers receive marginal product. The setting of Julien et al. is the limiting case of my model where $k()=$. and $\lambda / N=1$, so that every worker applies at all jobs. In the model of Albrecht et al. all workers send the same number of applications. Here, in contrast, the number of applications is stochastic, reflecting the role of chance in the search process. Workers do not decide at how many firms they apply, but rather how intensively they sample job openings. The model of Albrecht et al. also has an (irrelevant) wage posting stage prior to the application stage where firms commit to a lower wage bound which coincides in equilibrium with the reservation wage. My model has a different interpretation in that workers do not observe any job postings at the outset. Only after sampling, they apply to all suitable vacancies at zero cost. Therefore search in this model is random rather than directed. ${ }^{3}$

\subsection{The matching function}

Before solving the model, it is useful to consider the matching function of this model. Suppose, for the time being, that all workers decide the same search intensity $\lambda$ at stage II. For any worker $i$ the probability to get an offer from firm $j$, conditional on

\footnotetext{
${ }^{3}$ Nonetheless, in an extension of this model with heterogenous job types, workers might "direct search" by deciding how intensively they sample jobs of different types.
} 
$i$ applying at $j$, is $^{4}$

$$
z \equiv \frac{1-e^{-\lambda q}}{\lambda q}
$$

Hence, for any worker the probability to receive at least one offer (and thus to find a job) is

$$
\sum_{n \geq 1} \frac{1}{n !} \lambda^{n} e^{-\lambda}\left[1-(1-z)^{n}\right]=1-e^{-\lambda z}=1-e^{-\frac{1-e^{-\lambda q}}{q}} \equiv m(q, \lambda) .
$$

The matching rate for workers is increasing and concave in the job-worker ratio $1 / q$ (as usual), and it is strictly increasing in $\lambda$ : the more applications workers send on average, the more likely it is that every worker receives an offer. Such a result is not obvious; in fact it does not hold in the model of Albrecht, Gautier, and Vroman (2006) where the matching rate can be declining in the fixed (nonstochastic) number of applications. The reason for their result is that there are two coordination frictions with multiple applications. The first friction is based on lack of coordination between workers: some firms receive no applications while others receive multiple applications since workers do not coordinate at the application stage. The second friction is due to a lack of coordination between firms at the job offer stage: some workers do not receive any offer, others have multiple offers. Raising the number of applications mitigates the first friction but aggravates the second one: it becomes more likely that multiple firms contact the same worker. In my model the first effect always dominates so that the number of matches is globally increasing in the common search intensity.

When $\lambda \rightarrow \infty$, workers apply at all firms at stage I and the matching function is $m_{J}(q) \equiv 1-e^{-1 / q}$, the same as in the model of Julien, Kennes, and King (2000). In this limit only the second coordination friction is at work. In the model of Albrecht et al. (2006), the matching function is $m_{A}(q, a) \equiv 1-\left[1-\left(1-e^{-a q}\right) /(a q)\right]^{a}$ when all workers send $a$ applications; again the matching function of Julien et al. emerges as the special case $a \rightarrow \infty$. For finite $a$, it may be that $m_{A}(q, a)>m_{J}(q)$, so matching is more efficient with fewer applications. In my model, in contrast, matching is always more efficient the more applications are sent, i.e. $m(q, \lambda)<m_{J}(q)$ holds for finite $\lambda$. It can also be shown that $m(q, \lambda)<m_{A}(q, \lambda)$; matching is more efficient

\footnotetext{
${ }^{4}$ The derivation is standard: $\operatorname{Prob}(i$ gets offer from $j \mid i$ applies at $j) \operatorname{Prob}(i$ applies at $j)=z \cdot \lambda / N$ is equal to $\operatorname{Prob}(j$ gets $\geq 1$ appl. $) \operatorname{Prob}(i$ gets offer from $j \mid j$ gets $\geq 1$ appl. $)=\left(1-e^{-\lambda q}\right) \cdot 1 / M$. Solving yields $z$.
} 
when all workers send the same number of applications $a=\lambda$ than when they randomize applications from a Poisson distribution with mean $\lambda$.

\subsection{Equilibrium search intensity}

Consider the search intensity decision of workers at stage II after firm entry, so the worker-job ratio $q$ is given. A worker obtains income $p$ if he receives two or more offers at stage III, but he ends up with zero income otherwise. The probability to have two or more offers, conditional on $n$ applications, is

$$
1-(1-z)^{n}-n z(1-z)^{n-1}
$$

When the worker's search intensity is $\lambda$, the probability to end up with at least two offers is

$$
\sum_{n \geq 2} \frac{1}{n !} \lambda^{n} e^{-\lambda}\left[1-(1-z)^{n}-n z(1-z)^{n-1}\right]=1-e^{-\lambda z}(1+\lambda z) .
$$

When an individual worker in a large market decides $\lambda$, he takes $z$ (the probability to get an offer, conditional on applying) as given. Hence every worker solves

$$
\max _{\lambda \geq 0} U_{z}(\lambda) \equiv\left[1-e^{-\lambda z}(1+\lambda z)\right] p-k(\lambda)
$$

This objective function is typically not concave; for many cost functions it is convex at low values of $\lambda$ and concave at higher values. Moreover, $\lambda=0$ is always a local maximum when $k^{\prime}(0)>0$ holds. An interior (i.e. active search) local maximum must satisfy the first-order condition $U_{z}^{\prime}(\lambda)=0$, which is

$$
k^{\prime}(\lambda)=\lambda z^{2} e^{-\lambda z} p
$$

Whenever there exists a pure-strategy equilibrium where all workers choose the same search intensity $\lambda^{*}>0$, it follows from (1) and (3) that $\lambda^{*}$ solves

$$
k^{\prime}(\lambda)=\frac{\left(1-e^{-\lambda q}\right)^{2} e^{-\frac{1-e^{-\lambda q}}{q}}}{\lambda q^{2}} p
$$

To obtain analytical results, consider the uniformly elastic search cost function $k(\lambda)=k \lambda^{1+a} /(1+a)$ with $a \geq 0$ and $k>0$. Provided that the elasticity is large enough (i.e. the function is sufficiently convex), a unique pure strategy equilibrium must exist. 
Proposition 1: Let $k(\lambda)=k \lambda^{1+a} /(1+a)$ with $a>1$. Then, for any given $q$, there exists a unique equilibrium of the stage II subgame where all workers search with the same intensity $\lambda^{*}>0$ which is increasing in $p / k$.

Proof: Appendix.

When the cost function is not sufficiently convex, existence of a pure-strategy equilibrium with active search requires that labor market conditions are sufficiently good from the workers' perspective; that is, productivity must be high enough and there must be sufficiently many jobs per worker. Otherwise, the symmetric equilibrium is either one in "mixed strategies" where only a fraction of workers searches actively, or it is a no-activity equilibrium.

Proposition 2: Let $k(\lambda)=k \lambda^{1+a} /(1+a)$ with $a \in[0,1)$, and let $x$ be the unique positive solution of $e^{x}=1+x+x^{2} /(1+a)$. Further, define $\bar{q} \equiv \bar{z} \Phi(\bar{z}) / x$ where $\Phi(z)=\varphi$ is the inverse of $z=\left(1-e^{-\varphi}\right) / \varphi$, and $\bar{z} \equiv\left[\frac{k e^{x}}{p x^{1-a}}\right]^{1 /(1+a)}$. Then, for any given $q$, the unique equilibrium of the stage II subgame is as follows.

(a) If $p>k e^{x} / x^{1-a}$ and $q>\bar{q}$, a fraction $\alpha \in(0,1)$ of workers are active with search intensity $\lambda_{A}>0$ and fraction $1-\alpha$ of workers are inactive with $\lambda=0$.

(b) If $p>k e^{x} / x^{1-a}$ and $q \leq \bar{q}$, all workers search with the same intensity $\lambda^{*}>0$ which is increasing in $p / k$.

(c) If $p \leq k e^{x} / x^{1-a}$, all workers are inactive with $\lambda=0$.

Proof: Appendix.

When there are sufficiently many jobs per worker $(q \leq \bar{q})$, all workers decide to search with the same intensity. When this condition is violated, however, some workers cease to search at all, whilst others search with intensity $\lambda_{A}$. Workers must be indifferent between the search and the no-search strategies, so $U_{z}\left(\lambda_{A}\right)=0$ holds. This requirement together with the first-order condition $U_{z}^{\prime}\left(\lambda_{A}\right)=0$ determine the job-offer probability $z$ and search intensity for active workers $\lambda_{A}$. Therefore, these two numbers depend on productivity $p$ and on the search cost function, but they are independent of $q$. On the other hand, $z$ is related to the average search 
intensity $\lambda=\alpha \lambda^{A}$ according to equation (1). ${ }^{5}$ Let $\lambda q=\Phi(z)$ be the inverse of this relation, with $\Phi$ defined in Proposition 2. Then the fraction of active searchers is $\alpha=\Phi(z) /\left(q \lambda_{A}\right)$, which also shows that the number of active searchers per job $\alpha q$ is independent of market tightness $1 / q$. Put differently, any increase in job creation triggers a proportional increase in search activity. With the uniformly elastic search cost function, it is straightforward to obtain

$$
\lambda_{A}=\left[\frac{p x^{2}}{k e^{x}}\right]^{1 /(1+a)}, z=\left[\frac{k e^{x}}{p x^{1-a}}\right]^{1 /(1+a)}, \alpha=\frac{\Phi(z)}{q \lambda_{A}} .
$$

Corollary: In a mixed-strategy equilibrium, search intensity of active workers $\lambda_{A}$, the job-offer probability $z$ and the ratio of active workers per job $\alpha q$ are all independent of the worker-job ratio. The job-finding probability is $\alpha\left(1-e^{-\lambda_{A} z}\right)$, which increases proportionately with $1 / q$.

Although Proposition 2 is derived for a uniformly elastic cost function, I conjecture that results are similar for any arbitrary convex cost function with positive slope at $\lambda=0$ in which case the no-search strategy is a local maximum. All workers are active with the same search intensity when the labor market is tight (small $q$ ), whilst some workers are inactive when labor market prospects are less favorable from workers' perspective (large $q$ ). In the following, $\bar{q}$ denotes the threshold value of the worker-firm ratio separating an equilibrium with inactive workers from one without (where $\bar{q}=\infty$ is a possibility).

\section{$2.4 \quad$ Free entry}

To determine the endogenous number of jobs, note that a firm's profit is $p$ whenever it has at least one applicant and when the chosen applicant has no other offer. Otherwise profit is zero. When sufficiently many firms enter, there are no inactive workers and all workers search with the same intensity $\lambda^{*}(q)$. Expected profit is then

$$
\pi(q)=\left[1-e^{-q \lambda^{*}(q)}\right] e^{-\frac{1-e^{-q \lambda^{*}}(q)}{q}} p, q \leq \bar{q}
$$

\footnotetext{
${ }^{5}$ Note that $\alpha \lambda_{A} / N=\lambda / N$ is the probability that a given worker $i$ applies at a given firm $j$. Hence $\lambda$ is also the expected number of applications per worker and $z=\left(1-e^{-\lambda q}\right) /(\lambda q)$ is the probability of an offer, conditional on applying. The proof is the same as in footnote 4 .
} 
The expression in squared brackets is the probability that the firm has at least one applicant, and the second term is the probability that a randomly chosen applicant has no other offer. For fixed $\lambda^{*}$, profit is strictly increasing in $q$ : the larger the worker-job ratio, the more likely it is that a firm finds an applicant and the less likely it is that an applicant has multiple offers. When the effect of $q$ on $\lambda^{*}$ is taken into account, the overall impact of $q$ on $\pi$ is more complex since both the effect of $q$ on $\lambda^{*}$ and the one of $\lambda^{*}$ on $\pi$ are generally ambiguous. However, numerical experiments with the uniformly elastic cost function have shown that $\pi$ is strictly increasing in $q$ for arbitrary choices of the parameter $p / k$.

Conversely, when fewer firms enter, expected profit is

$$
\pi(q)=\left[1-e^{-\Phi(z)}\right] e^{-\lambda_{A} z} p, q>\bar{q} .
$$

Again the first expression is the probability to receive at least one application (since the number of applications at every firm is Poisson distributed with mean $\alpha \lambda_{A} q=$ $\Phi(z)$ ), and the second term is the probability that an active searcher gets no second offer, conditional on having one (since the number of job offers for active searchers is Poisson distributed with mean $\lambda_{A} z$ ). Importantly, expected profit in the range $q>\bar{q}$ does not depend on the worker-firm ratio $q$, since $z$ and $\lambda_{A}$ are independent of $q$ (note that this result does not depend on the functional form of the search cost function). In contrast to standard search models, more entry does not reduce the chance to find a worker since the number of active searchers increases proportionately with the number of jobs. For the same reason, the chance that a contacted worker has another other offer does not increase with the number of job openings.

The equilibrium worker-job ratio balances the cost for the marginal entrant to expected profit:

$$
\pi(q)=c(1 / q) .
$$

Whenever $c$ is strictly increasing with appropriate boundary conditions, there is a unique solution to this equation. To summarize, an equilibrium is a worker-firm ratio $q^{*}$ solving equation (7) together with the following search behavior of workers:

1. If $q^{*} \leq \bar{q}$, all workers search with common intensity $\lambda^{*}$ which is the larger solution to equation (4).

2. If $q^{*}>\bar{q}$, share $\alpha$ of workers search actively with intensity $\lambda_{A}$, while all others remain inactive. 


\subsection{Comparative statics}

Suppose that job surplus $p$ increases (for example, because productivity goes up or unemployment income falls). For a given number of firms, such a change has the following effects on search behavior. In a pure-strategy equilibrium, the common search intensity $\lambda^{*}$ increases unambiguously in $p$ (Proposition 1, and similarly in Proposition 2(b)). ${ }^{6}$ In a mixed-strategy equilibrium, both the number of active workers $\alpha$ and their search intensity $\lambda_{A}$ are increasing in $p$ (see equations (5)). Also the threshold value $\bar{q}$ increases; thus inactivity disappears when productivity is high enough. A larger job surplus raises the return to search, which unambiguously increases search activity and search intensity in this model for given $q$.

What is the effect of the productivity increase on job creation? The impact on firm profit in the range $q>\bar{q}$ is unambiguously positive: a larger $p$ raises the chance to find a worker (because more workers become active) and raises output in a filled job. In the range $q \leq \bar{q}$ the effect is less clear-cut. Although the chance to find a worker and job surplus go up again, the effect on the middle term in (6) is negative: the higher search intensity implies that workers are more likely to get a second offer in which case job profit would drop to zero. However, all my numerical experiments confirm that the overall impact of $p$ on firm profit is positive. Hence, an increase in productivity raises the job-to-worker ratio $1 / q$.

Figure 1 shows how job creation responds to such an increase in $p$, both in a purestrategy equilibrium where all workers are active and in a mixed-strategy equilibrium where some workers are inactive, for different levels of the entry cost function. In the regime without inactive workers $(q \leq \bar{q} \approx .55)$, the effect of a 20 percent increase of $p$ on $q$ is relatively modest: $q$ cannot fall by more than 10 percent, even when entry costs are perfectly inelastic. In the regime with inactive workers, however, the reaction of $q$ to an increase in $p$ can be large: since $\pi(q)$ is flat in the range $q>\bar{q}$, the elasticity of $q$ with respect to $p$ tends to infinity when the elasticity of $c$ with respect to $1 / q$ tends to zero.

\footnotetext{
${ }^{6}$ In this respect, the model differs from the one of Shimer (2004) where search intensity can also fall with higher job surplus.
} 


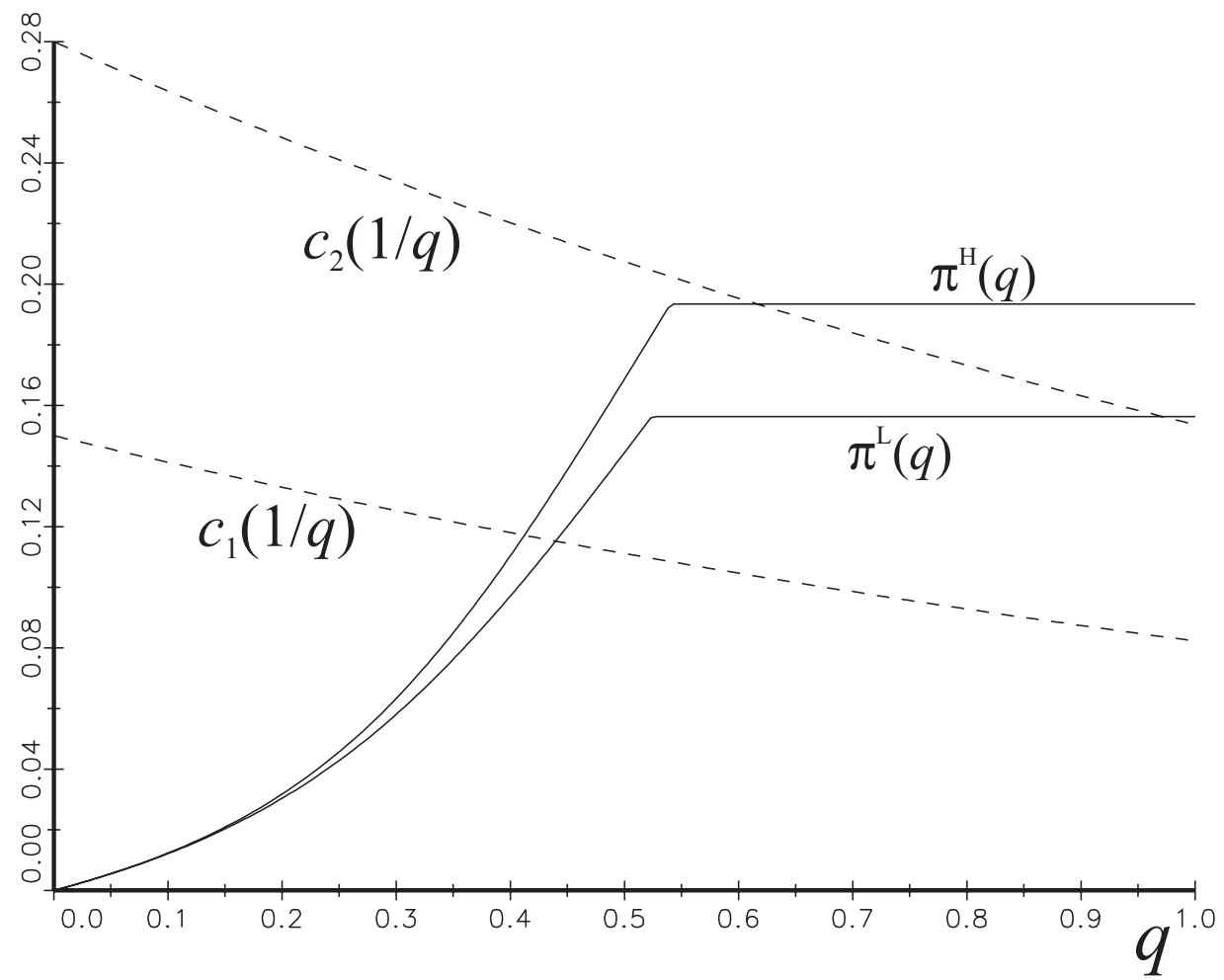

Figure 1: The response of the worker-firm ratio $q$ to a productivity increase from $p^{L}=1$ to $p^{H}=1.2$ with $k(\lambda)=.1 \cdot \lambda$ for two exemplary entry cost functions $c_{1}$ (pure-strategy equilibrium) and $c_{2}$ (mixed-strategy equilibrium).

\subsection{Efficiency}

In the model of Albrecht et al. (2006), the decentralized equilibrium is inefficient along two margins: entry is excessive and workers send too many applications. ${ }^{7}$ The first inefficiency also occurs in this model, but the second one must be qualified. In addition, another inefficiency emerges: it is never socially optimal that a fraction of workers remains inactive. To obtain these results, consider the problem of a social planner whose objective is to maximize total surplus per worker net of the costs of search and entry, with respect to $\lambda$ and $q$ :

$$
\max _{\lambda, q} m(q, \lambda) p-k(\lambda)-\int_{0}^{1 / q} c(v) d v
$$

\footnotetext{
${ }^{7}$ Albrecht et al. prove the first inefficiency only, but conduct numerical experiments for the second one.
} 
Observe first that the planner's objective is strictly concave in search intensity $\lambda$. Thus, it is never optimal to let fraction $\alpha \in(0,1)$ of workers search with positive intensity $\lambda_{A}$ while others are inactive. The planner rather prefers that all workers search with the same common intensity $\alpha \lambda_{A}$. Generally, the planner's objective depends on a distribution of search intensities, rather than a common intensity as it is written here. However, it is easy to show that nonparticipation of a fraction of workers is not optimal. ${ }^{8}$ The social optimum satisfies the following first-order conditions:

$$
\begin{aligned}
k^{\prime}(\lambda) & =e^{-\frac{1-e^{-\lambda q}}{q}} e^{-\lambda q} p \\
c(1 / q) & =e^{-\frac{1-e^{-\lambda q}}{q}}\left[1-(1+\lambda q) e^{-\lambda q}\right] p .
\end{aligned}
$$

Consider first the entry margin for a given common search intensity $\lambda$ of workers. In the decentralized equilibrium, the worker-firm ratio equates profit as in (6) to marginal entry cost $c(1 / q)$. On the other hand, the right-hand-side in the optimality condition (9) is strictly increasing in $q$ and smaller than profit $\pi(q)$. Hence, the worker-job ratio $q$ is too small in the decentralized equilibrium; entry is excessive. The explanation for the inefficiency is a similar "business-stealing effect" as it is discussed in Albrecht et al. (2006, p. 877): the social benefit of an additional vacancy falls short of the private benefit since the vacancy can attract workers from other firms whose vacancies are then left unfilled. Put differently, wages do not internalize the negative externality that an entrant exerts on incumbents who might lose all their applicants to the entrant firm. The inefficiency could go away if the wage for workers with one offer was greater than the monopsony wage (for example, if there is Nash bargaining with an appropriate choice of worker bargaining power). Obviously, in the limit $\lambda \rightarrow \infty$ this business-stealing effect disappears and entry becomes efficient, reconfirming the results of Julien et al. (2000).

Consider now the search intensity margin for a given worker-job ratio $q \leq \bar{q}$. Search intensity in the decentralized equilibrium is the larger solution to equation (4). The right-hand-side of the optimality condition (8) is declining in $\lambda$. Hence, equilibrium search intensity $\lambda^{*}$ is too large if and only if the right-hand-side of (4) is smaller

\footnotetext{
${ }^{8}$ Proof: in the mixed-strategy equilibrium, net surplus is $\alpha\left[m\left(\alpha q, \lambda_{A}\right) p-k\left(\lambda_{A}\right)\right]$, but if all workers search with intensity $\alpha \lambda_{A}$, surplus is $m\left(q, \alpha \lambda_{A}\right) p-k\left(\alpha \lambda_{A}\right)$. Weak convexity of $k$ implies $\alpha k\left(\lambda_{A}\right) \geq k\left(\alpha \lambda_{A}\right)$, and concavity of $1-e^{-x}$ implies that $\alpha m\left(\alpha q, \lambda_{A}\right)<m\left(q, \alpha \lambda_{A}\right)$.
} 
than the right-hand-side of (8) at $\lambda^{*}$ which is the same as

$$
\frac{\left(1-e^{-\lambda^{*} q}\right)^{2} e^{\lambda^{*} q}}{\lambda^{*} q^{2}}>1 .
$$

This inequality is true for all values of $q>0$ and $\lambda^{*} \geq 1$, but it may be violated when $\lambda^{*}<1$ and $q$ is not too large. When all workers send more than one application on average, search is socially excessive since workers impose a negative externality on other workers: because firms cannot coordinate their job offers, some workers receive no offers while others receive multiple offers. This externality is not internalized; on the contrary, workers desire to receive two or more offers, so the incentive to send a large number of applications is strong. Indeed, with a linear search cost function, $\lambda^{*}$ is always larger than $1 .^{9}$ In other situations, however, search intensity can also be inefficiently low. For instance, with $k(\lambda)=\lambda^{2} / 4, q=1$ and $p=1$, the equilibrium at $\lambda^{*}=.38$ is smaller than the social optimum at $\lambda=.65$.

Proposition 3: A mixed-strategy equilibrium where fraction $\alpha \in(0,1)$ of workers searches actively with intensity $\lambda_{A}$ is never socially optimal: welfare would increase if all workers searched with common intensity $\alpha \lambda_{A}$. A pure-strategy equilibrium $\left(q^{*}, \lambda^{*}\right)$ is not socially optimal since welfare can be raised by increasing $q$ at given $\lambda^{*}$. Moreover when $\lambda^{*} \geq 1$, welfare can be raised by lowering $\lambda$ at given $q^{*}$.

\section{Quantitative exploration}

\subsection{The matching function}

I will first demonstrate that this coordination-friction model with a reasonable choice of the period length and with fixed search intensity is unable to match the empirical response of the job-finding rate with respect to the vacancy-unemployment ratio. Any linearly homogeneous and concave matching technology gives rise to an increasing and concave relation between the vacancy-unemployment ratio $\theta=1 / q$ and the job-finding probability $\varphi(\theta)$. In my model this relation is given by equation (2), rewritten as

$$
\varphi(\theta)=1-e^{-\theta\left(1-e^{-\lambda / \theta}\right)} .
$$

\footnotetext{
${ }^{9}$ This follows from the second-order condition which requires that $\lambda z \geq 1$ and hence $\lambda \geq 1 / z>$ 1 .
} 
I choose the period length to be one week and set $\lambda$ to pin down the average weekly job-finding rate. A short period length is appropriate for this coordination friction model (as for the others discussed below) since workers send applications only once in a period and since firms can contact only one worker per period.

Robert Shimer calculates the average monthly job-finding rate in postwar U.S. data to be around $0.45 .{ }^{10}$ On a weekly basis, this leads to $\varphi=1-.55^{1 / 4.35} \approx 0.128$. As in Hagedorn and Manovskii (2007), the mean vacancy-unemployment ratio is set at $\theta=0.634$ in order to be consistent with Den Haan, Ramey and Watson's (2000) estimate of the mean job-filling rate. ${ }^{11}$ These numbers imply that $\lambda=0.155$. Hence, an average unemployed worker sends one application within 6.5 weeks and gets a job after after applying with a chance of $z=\left(1-e^{-\lambda q}\right) /(\lambda q)=88.7 \%$. The first number seems too small and the second one too large. However, the consideration of an (irrelevant) mismatch parameter can help explain these magnitudes. Suppose workers sends $\lambda_{0}$ applications on average, and that the firm observes match-specific productivity after it receives the application. With probability $\mu$ the worker fits the job, but with probability $1-\mu$ the worker's productivity is too low for the firm to be willing to offer the position. Then each worker sends only $\lambda=\mu \lambda_{0}$ "effective applications" (those that result in productive matches) which may be much smaller than the average number of actual applications $\lambda_{0}$. Similarly, $\lambda q$ is the average number of effective applications per vacancy, and $z$ is the offer probability for an effective application. The actual number of applications and the probability to draw high match-specific productivity are irrelevant for the quantitative analysis. All that matters are the effective application rates.

The main result of this analysis is that the elasticity of the matching function is too low compared to empirical measures. Shimer (2005) estimates the elasticity of the job-finding rate with respect to the vacancy-unemployment ratio to be 0.28 ; Petrongolo and Pissarides (2001) conclude in their survey that the plausible range for this elasticity is the interval $[0.3,0.5]$. With my matching technology and the empirical values for $\theta$ and $\lambda$ (targeting the average $\varphi$ ), I obtain $\varepsilon_{\varphi, \theta}=.109$, clearly below the range of plausible values. ${ }^{12}$

\footnotetext{
${ }^{10}$ See Shimer (2007b) and http://robert.shimer.googlepages.com/flows

${ }^{11}$ All quantitative results are similar with $\theta=0.454$ which is obtained from the direct vacancy measure of the Job Opening and Labor Turnover Survey for the period 12/2007-07/2007.

${ }^{12}$ This result critically depends on the choice of the period length. With a 14 -day period, the elasticity roughly doubles but is still too low. Nonetheless, a short period length is reasonable
} 
The result that the elasticity of the matching function is too low is not only true in my model but in virtually all established coordination-friction models of the literature. That is, the basic versions of such models, with homogenous agents, with a reasonable choice of the period length, and without variable search intensity, are unable to match the empirical elasticity of the matching function. To see this, consider the matching function of Albrecht, Gautier, and Vroman (2006) which is general enough to encompass those of most other models as special cases. In their model, every worker sends $a$ applications, randomly (in equilibrium) to homogenous firms. After applications arrived, each firm makes a job offer to at most one worker, rejecting all others. The model gives rise to the job-finding rate

$$
\varphi(\theta)=1-[1-z(a / \theta)]^{a}
$$

where $z(x)=\left(1-e^{-x}\right) / x$ is the probability to receive an offer. The large-economy model of Montgomery (1991) and Burdett, Shi, and Wright (2001) is the special case $a=1$, and the model of Julien, Kennes, and King (2000) is the limit $a \rightarrow \infty$. For given $a$ ("fixed search intensity"), this matching function is not flexible enough to match the job-finding rate at the empirical mean value of $\theta$, since $\theta$ is the only free parameter. However, match-specific productivity enriches the model sufficiently to achieve this requirement (see also Petrongolo and Pissarides (2001)). ${ }^{13}$ When the number of actual applications is a given parameter, match-specific productivity can be used to target the job-finding rate. As above, suppose firms learn what applicants are suitable after they receive applications and let $\mu$ be the probability that a worker fits the job. With this modification, the job-finding rate becomes

$$
\varphi_{\mu}(\theta)=1-[1-\mu z(a \mu / \theta)]^{a} .
$$

When I use the parameter $\mu$ to target the weekly job-finding rate at given $\theta=0.634$, I find that $\mu=0.143(a=1), \mu=0.0742(a=2), \mu=0.0153(a=10)$; hence the expected number of effective applications $a \mu$ is nearly constant and about the same as my calibrated value for $\lambda$. With fixed search intensity $a$, the job-finding rate

for this microfoundation of matching frictions. Most newspapers and job agencies publish job advertisements at weekly or even higher frequency. It seems implausible that job searchers sample such job openings at a frequency which is much longer than a week.

${ }^{13}$ Alternatively, one could try to use the number of applications $a$ as a free parameter. However, to match $\varphi=0.124$ at $\theta=0.639$ requires $a=.0374$, a number which is not compatible with this model which requires $a \in \mathbb{N}$. 
responds to little to variations in the vacancy-unemployment ratio. I compute an elasticity of the job-finding rate with respect to the vacancy-unemployment ratio of 0.1085 (for $a=1$ ) and 0.1089 (for $a=100$ ), clearly below the range of plausible values and about the same as with my matching function.

These results suggest that other mechanisms are required in order to match coordinationfriction economies to the data. In the following, I consider a dynamic extension of the static model in which parameters of a reduced-form search cost function are calibrated to match the mean and elasticity of the job-finding rate. The purpose of this study is to answer the following questions.

- How large and how volatile must search costs be to be consistent with the data?

- What are the implications of variable search intensity for the amplification of shocks?

- What are the welfare implications?

\subsection{The dynamic model}

I embed the static model of Section 2 in a dynamic framework in discrete time $t$. The environment is stationary, so I consider a steady state equilibrium and confine the quantitative analysis to comparative statics experiments. Since actual productivity shocks are quite persistent and since adjustment to the steady state is fast, the comparative statics response should be a reasonable approximation to the dynamic response (see also Mortensen (2007)).

Existing jobs end with exogenous probability $\delta$ per period. There is no search on the job, so $q$ is the number of unemployed workers per vacancy. Flow output in a filled job is $p$ and flow unemployment income is $b$. This term is interpreted to encompass unemployment benefits, income from home production, and the utility value of leisure (in excess of an employed person), but it does not include the cost of job search. Workers want to maximize their expected utility

$$
E \sum_{t=0}^{\infty} \beta^{t}\left[w_{t}-k\left(\lambda_{t}\right)\right],
$$


where $\beta$ is the discount factor, $w_{t}$ is wage or unemployment income in period $t$, and $k(\lambda)=k \cdot \lambda^{1+a} /(1+a)$ is the utility cost of job search at search intensity $\lambda$, with $a \geq 0$ and $k>0$. The search cost function is a shortcut to capture both the opportunity cost of foregone leisure time and the hedonic cost of job search. ${ }^{14}$ Within every period, unemployed workers and vacant jobs are matched according to the same process as in the static model. Unmatched agents continue search in the next period and there is no recall.

I restrict attention to a pure-strategy equilibrium where all nonemployed workers are active searchers (so they are classified as unemployed in the usual definition). It is straightforward to characterize mixed-strategy equilibria with inactive workers, but they do not deliver reasonable predictions in the numerical experiments. Specifically, in comparison with U.S. data, inactivity (i.e. nonemployed persons who want to work but do not search) becomes too volatile relative to unemployment, and vacancies vary too little relative to nonemployment (unemployment plus inactivity). Also the predictions of the corollary after Proposition 2 are at odds with the evidence. Hence the inactivity mechanism of this model can only be quantitatively relevant when it is coupled with substantial heterogeneity.

As in the static model, there are jobs with high and low wages, depending on the number of offers a worker holds in his hand when leaving unemployment. Although low-wage earners may have an incentive to search for better-paid jobs, I assume that employers can identify the current employment status of an applicant and that employed workers can renegotiate when they obtain better offers later on. Under these assumptions, employers will never offer their vacant job to an employed worker, and search on the job does not take place. ${ }^{15}$

Let $V$ and $J^{j}$ be the values of vacant and filled jobs with wages $w^{j}$ in job status $j=l, h$. Suppose that flow costs of a vacant job are $c$, independent of the number

\footnotetext{
${ }^{14}$ An alternative strategy (avoiding a reduced-form search cost function but ignoring hedonic search costs) would be to include leisure time in the utility function and to calibrate the parameters of this function using the empirical Frisch elasticity and time-use data on work and job search. However, since the functional relationship between the observable "search time" and the unobservable "search intensity" $\lambda$ is unclear, I refrained from pursuing this approach.

${ }^{15}$ This assumption is similar as in Julien, Kennes, and King (2006). In their model, however, low-wage earners search on the job, but only because of between-firm productivity differentials, which are absent here.
} 
of active firms. The Bellman equations are

$$
\begin{aligned}
V & =-c+\beta V+\left(1-e^{-\lambda q}\right) e^{-\lambda z} \beta\left(J^{l}-V\right), \\
J^{l} & =p-w^{l}+\beta(1-\delta) J^{l} \\
J^{h} & =p-w^{h}+\beta(1-\delta) J^{h} .
\end{aligned}
$$

In the first equation, firms gain only when they meet an applicant with no other offer, which happens with probability $\left(1-e^{-\lambda q}\right) e^{-\lambda z}$. Otherwise they either get no application or they offer the job to a worker who has at least one other offer. In this case, Bertrand competition drives the wage $w^{h}$ so high that the value of the filled job is equal to the value of a vacant job, which is zero, so it follows that $w^{h}=p$. The free-entry condition $V=0$ is

$$
c=\left(1-e^{-\lambda q}\right) e^{-\lambda z} \frac{\beta\left(p-w^{l}\right)}{1-\beta(1-\delta)} .
$$

For workers, let $U$ and $E^{j}$ be the utility value when the current state is unemployment or employment in a job of status $j=l, h$. Workers' Bellman equations are

$$
\begin{aligned}
U & =b+\beta U+\max _{\lambda \geq 0}\left[\gamma(\lambda z) \beta\left(E^{h}-U\right)-k(\lambda)\right], \\
E^{l} & =w^{l}+\beta E^{l}+\beta \delta\left(U-E^{l}\right), \\
E^{h} & =p+\beta E^{h}+\beta \delta\left(U-E^{h}\right) .
\end{aligned}
$$

Here, $\gamma(x)=1-(1+x) e^{-x}$ is the probability to find a high-wage job (that is, to obtain two or more job offers) for a worker who receives $x=\lambda z$ offers in expectation. Since workers with only one offer do not gain at the transition from unemployment to employment, $E^{l}=U$ must hold, which gives rise to the reservation wage equation

$$
w^{l}=b-k(\lambda)+\beta \gamma(\lambda z)\left(E^{h}-U\right) .
$$

The utility increase at a transition into a high-wage job is

$$
E^{h}-U=\frac{p-w^{l}}{1-\beta(1-\delta)} .
$$

As in the static model, optimal search intensity equates marginal search costs to the marginal return from search:

$$
k \lambda^{a}=\lambda z^{2} e^{-\lambda z} \beta\left(E^{h}-U\right) .
$$

A steady-state equilibrium is a vector $\left(z^{*}, \lambda^{*}, w^{l *},\left(E^{h}-U\right)^{*}, q^{*}\right)$ solving the five equations (1), (11), (12), (13), and (14). 


\subsection{Calibration}

There are seven parameters $(\beta, \delta, a, k, c, b, p)$ which are calibrated to the U.S. economy. In comparison with calibration exercises of standard Mortensen-Pissarides models, the two parameters of the aggregate matching function (a level and an elasticity parameter) are replaced by the two parameters $a$ and $k$ of the reduced-form search cost function and they play a similar role, targeting the mean and the elasticity of the job-finding rate. Because wages are determined by an ex-post auction, there is no wage bargaining parameter. Although I could easily augment the model by Nash bargaining in those matches where a worker has no alternative offers, the result would be that wages react too much to productivity (see the discussion in 3.4 below).

As above, I specify the period length to be one week. With an annual interest rate of $5 \%$, this implies $\beta=.9991$. With an average weekly job-finding rate of $\varphi=.128$ and an average unemployment rate of $5.6 \%$, the weekly separation rate is fixed at $\delta=0.762 \%$ which is consistent with Shimer's (2005) measure of the monthly separation rate of $2.6 \%$ (after adjusting for time aggregation).

Productivity is normalized at $p=1$, and vacancy costs are set at Hagedorn and Manovskii's (2008) estimated value of $c=0.58$, though I do not allow for a productivity dependence, as they do. The remaining parameters $b, k$ and $a$ are pinned down to match the following three targets; the mean job-finding rate of $\varphi=0.128$, the mean vacancy-unemployment ratio $\theta=1 / q=0.634$, and the elasticity of the job-finding rate with respect to $\theta$ at $\varepsilon_{\varphi, \theta}=0.28$ (see Section 3.1 for explanations), provided that variation in the steady-state value of $\theta$ is driven by a permanent productivity shift. In principle, $\lambda$ (and thus $\varphi$ ) reacts to a shock for two reasons: job surplus $E^{h}-U$ changes and the job-offer probability $z$ changes. How much job surplus changes relative to $z$ depends of course on the source of the shock. However, $\varepsilon_{\varphi, \theta}$ does not change much when variation in $\theta$ is driven by shocks to the separation rate or to unemployment income. More precisely, when parameter $a$ targets $\varepsilon_{\varphi, \theta}=0.28$ for a $p$ shock, it can be verified that then $\varepsilon_{\varphi, \theta}=0.2804$ (with a $b$ shock) and $\varepsilon_{\varphi, \theta}=0.2803$ (with a $\delta$ shock).

The calibration targets imply that $b=0.955, k=6051.6$ and $a=5.27 .{ }^{16}$ As in

\footnotetext{
${ }^{16}$ Numerically, for given $a$ and the targets for $\varphi$ and $\theta$ (and hence $\lambda$ and $z$ ), equation (11) yields $w^{l}, k$ follows from (13) and (14) and $b$ follows from (12). Then $a$ is adjusted to target $\varepsilon_{\varphi, \theta}$.
} 
Section 3.1, mean search intensity is at $\lambda=0.155$ and the chance to obtain an offer is $z=0.887$. Mean search costs are modest at $k(\lambda)=0.0081$, so that the flow utility from unemployment is at $\hat{b}=b-k(\lambda)=0.947$.

The reservation wage is at $w^{l}=0.9738$, so that the mean wage is at $\bar{w}=\alpha w^{l}+1-\alpha=$ 0.9756 , where $\alpha=\lambda z e^{-\lambda z} /\left(1-e^{-\lambda z}\right)=0.933$ is the share of workers in low-wage jobs. Finally, because of $a>1$ and Proposition 1, the first-order condition (14) indeed characterizes the unique equilibrium in pure strategies. Table 1 summarizes parameter choices and their explanation.

\begin{tabular}{|r|r|l|}
\hline Parameter & Value & Explanation \\
\hline$\beta$ & 0.9991 & Annual interest rate $5 \%$ \\
$\delta$ & 0.00762 & Unemployment rate $u=5.6 \%$ \\
$p$ & 1 & Normalization \\
$c$ & 0.58 & Hagedorn and Manovskii $(2008)$ \\
$k$ & 6051.6 & Job-finding rate $\varphi=0.128($ via $\lambda)$ \\
$b$ & 0.955 & Vacancy-unemployment ratio $\theta=0.634\left(\right.$ via $w^{l}$ and $\left.J^{l}\right)$ \\
$a$ & 5.27 & Matching-function elasticity $\varepsilon_{\varphi, \theta}=0.28$ \\
\hline
\end{tabular}

Table 1: Parameter choices.

\subsection{Quantitative implications}

How does the economy respond to a permanent unanticipated productivity increase? As implied by Proposition 1, search intensity will go up. Indeed, at the calibrated parameter values, I calculate elasticities $\varepsilon_{\lambda, p}=4.7$ and $\varepsilon_{k(\lambda), p}=29.7$. Although search costs are small (only $0.8 \%$ of unemployment utility), they are quite volatile, varying between $0.3 \%$ and $1.3 \%$ when $p$ varies between 0.98 and 1.02. Empirical results on the response of search intensity to market conditions are inconclusive. The findings of Shimer (2004) do not support procyclical search intensity. He considers CPS data and uses two measures of search intensity. One is the "probability to search" which is irrelevant here since nonparticipation is not considered. The other is the "number of job search methods". However, the variability of this measure is small and it is unclear how this number correlates with application rates and with search time, which are relevant here. On the other hand, Krueger and Mueller 
(2008) examine time-use data and find that job-search time responds positively to labor market conditions. Using cross-state variation in unemployment benefits, they calculate an elasticity of job search with respect to benefits of around -2 . My model yields the elasticity $\varepsilon_{\lambda, b_{0}}=-1.4$, where $b_{0}=.28=.29 \bar{w}$ is a plausible value for the level of unemployment benefits. ${ }^{17}$ If search intensity $\lambda$ is proportional to job-search time, this finding suggests that my calibration does not overstate the responsiveness of search intensity to labor market conditions. Krueger and Mueller also find that an individual's predicted wage has a strong quantitative impact on job-search time. It is unsurprising that the model's response of the vacancy-unemployment ratio to a productivity shock is of the same magnitude as in the data. In fact, my level of flow unemployment income is about the same as in the calibration of Hagedorn and Manovskii (2007) which is well known to yield a strong amplification. This is even true when search intensity is fixed at the steady-state value, in which case I find that $\varepsilon_{\theta, p}^{\lambda \text { fixed }}=18.8$, about 10 times as large as in Shimer's (2005) calibration of the search and matching model where flow unemployment income is at $\hat{b}=0.4$. When search intensity varies positively with productivity, the amplification of productivity is even stronger since the job-finding rate increases and hence unemployment decreases further. However, the impact on the elasticity is modest; it merely increases to $\varepsilon_{\theta, p}^{\lambda}$ variable $=22.9$, so the order of magnitude does not change. My calibration also gives rise to a reasonable response of wages. I calculate an elasticity of the mean wage with respect to productivity of $\varepsilon_{\bar{w}, p}=0.61$. This number is a bit larger than the estimate of $\varepsilon_{w, p}=0.45$ used by Hagedorn and Manovskii to pin down the bargaining power parameter in the Mortensen-Pissarides model. Again, this is not surprising. In their calibration, workers get $5.2 \%$ of job surplus, whereas here $6.7 \%$ of workers obtain the full job surplus whilst the rest works at the reservation wage, obtaining zero surplus. Therefore the wage response in my model is somewhat stronger, and this is the reason why it makes little sense to augment the model by Nash bargaining in one-one matches.

It is instructive to see what happens when the level of unemployment utility is reduced to the value used by Shimer (2005) in his calibration of the search-andmatching model. To this end, I choose $b$ to obtain in steady state $\hat{b}=b-k(\lambda)=0.4$. In the absence of a Nash bargaining parameter, it is clear that this choice blows up profits, so I set now $c=6.61$ to still target the steady-state vacancy-unemployment

\footnotetext{
${ }^{17}$ See Nickell, Nunziata, and Ochel (2005) who calculate a replacement ratio for the U.S. of $29 \%$.
} 
ratio at $\theta=0.634$. Again $a$ is chosen to target $\varepsilon_{\varphi, \theta}=0.28$, and $k$ is chosen to target $\varphi=0.128$. Interestingly, the elasticity of search costs $a=5.27$ is the same as before, but $k$ is about one order of magnitude larger. Hence I find that search costs are $k(\lambda)=.092$, now more than $20 \%$ of unemployment utility. On the other hand, the responsiveness of search intensity (and search costs) are reduced by an order of magnitude; that is $\varepsilon_{\lambda, p}=0.416$. As with the original parameter values, variable search intensity raises the responsiveness of the vacancy-unemployment ratio only slightly. Specifically, $\varepsilon_{\theta, p}$ increases from 1.65 with fixed intensity to 2.01 with variable search intensity.

\subsection{Welfare}

Results from section 2.6. show that equilibrium is not efficient in general: entry is excessive and search intensity can be too high or too low. The dynamic model requires a separate analysis of the welfare issue. One important adjustment concerns the role of unemployment benefits which are pure transfers and do not contribute to welfare. ${ }^{18}$ However, benefits induce a wedge between the private and the social job surplus, which dampens the incentives to search for workers and firms. This effect counteracts my previous findings that entry is excessive and that search intensity is excessive when $\lambda>1$.

Because of quasilinear preferences, the planner's objective is to maximize a utilitarian welfare function which adds up the discounted value of the income stream net of entry cost and worker's search effort costs. The recursive formulation of this problem is

$$
\begin{array}{r}
W(u)=\max _{\lambda, q}\left\{u \cdot[\tilde{b}-k(\lambda)]+(1-u) \cdot p-\frac{c u}{q}+\beta W\left(u^{\prime}\right)\right. \\
\text { s.t. } \left.u^{\prime}=u+\delta(1-u)-m(q, \lambda) u\right\} .
\end{array}
$$

Here $u$ is the unemployment rate (the only state variable), $W(u)$ is welfare when current unemployment is $u, m(q, \lambda)$ is the matching function $(2)$, and $\tilde{b}=b-b_{0}$ is

\footnotetext{
${ }^{18}$ Of course, matters would be different if workers were risk averse and markets were incomplete. In their welfare analysis, Hagedorn and Manovskii (2008) correct for distortionary taxes, but they ignore any positive role of government.
} 
flow unemployment utility net of benefits $b_{0}$ and gross of search costs. I obtain the following characterization of the social optimum.

Proposition 4: In the social optimum, the unemployment-vacancy ratio $q$ and search intensity $\lambda$ are independent of the state variable $u$ and satisfy the first-order conditions

$$
\begin{aligned}
c & =\beta e^{-\lambda z}\left[1-(1+\lambda q) e^{-\lambda q}\right] S, \\
k^{\prime}(\lambda) & =\beta e^{-\lambda z} e^{-\lambda q} S,
\end{aligned}
$$

where $z=\left(1-e^{-\lambda q}\right) /(\lambda q)$ and

$$
S=\frac{p-\tilde{b}+k(\lambda)}{1+\beta \delta-\beta e^{-\lambda z}(1+\lambda z)+\beta \lambda e^{-\lambda z} e^{-\lambda q}}
$$

is the social value of an employed person.

Proof: Appendix.

The intuition behind the optimality conditions is easy to explain. In (16), the term $e^{-\lambda z}\left[1-(1+\lambda q) e^{-\lambda q}\right]$ is the same as $d[m(q, \lambda)] / d[1 / q]$, i.e. the number of new matches of an additional vacancy. Hence the term on the right-hand side is the social return of a vacancy which must be equal to marginal cost on the left-hand side. Similarly in (17), the term $e^{-\lambda z} e^{-\lambda q}$ is the same as $d[m(q, \lambda)] / d[\lambda]$, so the right-hand side is the marginal social return of an additional unit of search intensity.

When there are no unemployment benefits, it is straightforward to show that entry is excessive. Indeed, the free-entry condition (11), using (12) and (13), can be expressed as

$$
c=\beta e^{-\lambda z}\left(1-e^{-\lambda q}\right) \frac{p-b+k(\lambda)}{1+\beta \delta-\beta e^{-\lambda z}(1+\lambda z)} .
$$

It follows immediately that the right-hand side of (16) is smaller than the righthand side of (18), at the same values of $\lambda$ and $q$ when $b=\tilde{b}$. Since the right-hand side of (16) is increasing in $q$, the equilibrium level of $q$ is smaller than the socially optimal level of $q$ (at given $\lambda$ ). Hence, for any given level of search intensity, there is too much entry. Obviously, this result can change when benefits $b-\tilde{b}>0$ are large enough.

Table 2 compares the equilibrium at the benchmark calibration with the social optimum, where I set benefits to $b_{0}=.28$, at $29 \%$ of the mean wage (see footnote 17 ). 
Compared with the social optimum, equilibrium search intensity is about 25 percent too low, and the vacancy-unemployment ratio $1 / q$ is more than twice as large. The planner would choose about the same unemployment rate, but the optimal vacancy rate would be about half of what is in the data. Nonetheless, total welfare $W(u)$ is only about $1 \%$ below optimum. The last three columns of the table show the three components of flow surplus: output, flow utility of the unemployed, and vacancy costs. The planner would like to reduce unemployment utility slightly (by inducing them to search more) and create fewer vacancies; actual output (employment) would increase only minimally.

\begin{tabular}{|l|l|l|l|l|l|l|l|}
\hline & $\lambda$ & $q$ & $u(\%)$ & Welfare & $\begin{array}{l}\text { Flow } \\
\text { output }\end{array}$ & $\begin{array}{l}\text { Flow utility of } \\
\text { unemployed }\end{array}$ & $\begin{array}{l}\text { Vacancy } \\
\text { costs }\end{array}$ \\
\hline Equilibrium & 0.155 & 1.577 & 5.6 & 1063.7 & 0.944 & 0.037 & 0.021 \\
Social opt. & 0.207 & 3.712 & 5.36 & 1075.6 & 0.946 & 0.033 & 0.008 \\
\hline
\end{tabular}

Table 2: Equilibrium versus social optimum.

\section{Conclusion}

This paper develops a model of coordination frictions with variable search intensity in which equilibrium can be fully characterized by a simple set of first-order conditions. Under bad labor market conditions, there can be an equilibrium where only a fraction of workers are active searchers whilst the rest stays out of the labor market. This inactivity phenomenon is not socially desirable; the planner desires that all homogenous workers search with a common intensity. But even under better labor market conditions, equilibrium is typically inefficient, as there are too many vacancies and a suboptimal level of search intensity. In the quantitative part of this paper, I argue that variable search intensity helps to improve the fit of coordinationfriction models which typically have a too low matching-function elasticity, and I examine the comparative statics features of a reasonably calibrated dynamic model. Of course, variable search intensity may not be the only mechanism raising the responsiveness of the job-finding rate. Lester (2008) shows that an endogenous capacity choice in the model of Burdett, Shi and Wright (2001) can also raise the elasticity of the job-finding rate with respect to productivity. 
The following two extensions are left for future work. The first is the effect of heterogeneity on search intensities. On the one hand, when there are jobs with high and low productivities, it is interesting to know whether workers search too hard for the good jobs and too little for the bad ones, and how much they diversify their "search portfolio". A similar issue is analyzed in Gautier and Wolthoff (2007). On the other hand, when workers are endowed with different skills, it should be understood how much search intensities between skill groups differ, and what the implications are for skill differences in wages and employment rates.

Another variation concerns the wage determination mechanism. If firms commit to posted wages and workers apply after sampling these postings, there should be wage dispersion similarly as in the model of Burdett and Judd (1983). Such a model can also be examined when firms are able to recall all their applicants as in Kircher's (2007) model.

\section{Appendix}

\section{Proof of Proposition 1:}

Consider the best response problem of a particular worker deciding search intensity $\lambda$ when all others search with intensity $\lambda_{0}$. Let $z=z\left(\lambda_{0}\right)=\left[1-e^{-\lambda_{0} q}\right] /\left(\lambda_{0} q\right)$ be the chance to receive an offer when applying. The worker's best response is the solution to $U_{z}^{\prime}(\lambda)=0$ which is

$$
z^{2} e^{-\lambda z} \frac{p}{k}=\lambda^{a-1}
$$

Because of $a>1$, this equation has a unique solution which is the global maximum of $U_{z}($.$) . Hence, for any \lambda_{0}$ there is a unique best response, denoted $\lambda=S\left(z\left(\lambda_{0}\right)\right) \equiv$ $R\left(\lambda_{0}\right)$. Moreover, because of $z(0)=1$ and $z(\infty)=0, R(0)>0$ and $R(\infty)=0$. Since $R$ is continuous, a Nash equilibrium $\lambda=R(\lambda)>0$ exists.

To prove uniqueness, it suffices to show that $R^{\prime}(\lambda)=S^{\prime}[z(\lambda)] z^{\prime}(\lambda)<1$ at any $\lambda=R(\lambda)$. One has

$$
z^{\prime}(\lambda)=-\frac{1-e^{-\lambda q}(1+\lambda q)}{\lambda^{2} q},
$$

and implicit differentiation of (19) yields

$$
S^{\prime}(z)=\frac{\lambda(2-\lambda z)}{\lambda z^{2}+(a-1) z} .
$$


Combining these two shows that $R^{\prime}(\lambda)<1$ (at any $\lambda=R(\lambda)$ ) is equivalent to

$$
-\frac{(2-\lambda z)\left[1-e^{-\lambda q}(1+\lambda q)\right]}{\left[\lambda z^{2}+(a-1) z\right] \lambda q}<1 .
$$

With $a>1$, a sufficient condition for this inequality is that

$$
-(2-\lambda z)\left[1-e^{-\lambda q}(1+\lambda q)\right]<z^{2} \lambda^{2} q .
$$

Substitution of $z=\left(1-e^{-\lambda q}\right) /(\lambda q)$ obtains the equivalent

$$
2 e^{\lambda q}-2-2 \lambda q>\lambda e^{-\lambda q}-\lambda .
$$

This inequality is satisfied since the right-hand side is negative and since $e^{x}>1+x$.

Lastly, the unique Nash equilibrium $\lambda^{*}$ increases in $p / k$ because the best-response curve $R($.$) shifts upwards when p / k$ increases, which follows immediately from (19) defining $\lambda=S(z)$.

\section{Proof of Proposition 2:}

Consider again the best-response problem of a particular worker when $\lambda_{0}$ is the average search intensity of other workers (who either all search with intensity $\lambda_{0}$, or fraction $\alpha$ search with intensity $\left.\lambda_{A}=\lambda_{0} / \alpha\right)$. As in the proof of Proposition $1, z=z\left(\lambda_{0}\right)$ is the job-offer probability conditional on applying. The worker's objective function $U_{z}(\lambda)$ has an interior local maximum whenever the increasing, strictly concave $\lambda^{a}$ has two positive intersections with the hump-shaped $\lambda z^{2} e^{-\lambda z} p / k$. Such intersections exist if and only if

$$
\frac{p}{k}>e^{1-a}(1-a)^{a-1} z^{-1-a} .
$$

This is the same as

$$
z=z\left(\lambda_{0}\right)>\hat{z} \equiv\left[\frac{k e^{1-a}}{p(1-a)^{1-a}}\right]^{1 /(1+a)},
$$

which is the same as

$$
\lambda_{0}<\hat{\lambda}_{0} \equiv \frac{1}{q} \Phi(\hat{z}) .
$$

Therefore, for any $\lambda_{0}<\hat{\lambda}_{0}, U_{z}(\lambda)$ has an interior local maximum, to be denoted $\lambda=R\left(\lambda_{0}\right)>0$. Worker payoff at the local maximum is then

$$
\begin{aligned}
U_{z}(\lambda) & =p\left[1-e^{-\lambda z}(1+\lambda z)\right]-\frac{k}{1+a} \lambda^{1+a} \\
& =p e^{-\lambda z}\left[e^{\lambda z}-1-\lambda z-\frac{(\lambda z)^{2}}{1+a}\right] .
\end{aligned}
$$


Therefore, $\lambda=R\left(\lambda_{0}\right)$ is a global maximum (that is, $U_{z}(\lambda) \geq 0$ so that a deviation to $\lambda=0$ does not raise payoff) if and only if

$$
\lambda z \geq x
$$

where $x$ is the unique positive solution of

$$
e^{x}=1+x+\frac{x^{2}}{1+a} .
$$

Since $\lambda=R\left(\lambda_{0}\right)$ solves $\lambda^{a}=\lambda z^{2} e^{-\lambda z} p / k$,

$$
\frac{e^{\lambda z}}{(\lambda z)^{1-a}} \frac{k}{p}=z^{1+a}
$$

holds. Because $e^{\lambda z} /(\lambda z)^{1-a}$ is increasing in $\lambda z \geq 1-a$ (where $\lambda z>1-a$ follows from the second--order condition $U_{z}^{\prime \prime}(\lambda)<0$ ), condition $(21)$ is equivalent to

$$
\frac{e^{x}}{x^{1-a}} \frac{k}{p} \leq z^{1+a}
$$

or

$$
z=z\left(\lambda_{0}\right) \geq \bar{z} \equiv\left[\frac{k e^{x}}{p x^{1-a}}\right]^{1 /(1+a)} .
$$

Therefore, $\lambda=R\left(\lambda_{0}\right)>0$ is best response to $\lambda_{0}$ iff

$$
\lambda_{0} \leq \bar{\lambda}_{0} \equiv \frac{1}{q} \Phi(\bar{z})
$$

and it is the unique best response if the inequality is strict. Note also that $\bar{\lambda}_{0}>\hat{\lambda}_{0}$. Further, for any $\lambda_{0} \geq \bar{\lambda}_{0}, \lambda=0$ is best response to $\lambda_{0}$, and it is the unique best response when the inequality is strict.

Since $\bar{\lambda}_{0} \leq 0$ is equivalent to $\bar{z} \geq 1, \lambda=0$ is the unique Nash equilibrium when $p \leq$ $k e^{x} / x^{1-a}$ (part (c) of the Proposition). When $p>k e^{x} / x^{1-a}$ is satisfied, $\bar{\lambda}_{0}>0$ holds. In that case, a pure-strategy equilibrium exists when $R\left(\bar{\lambda}_{0}\right) \leq \bar{\lambda}_{0}$ (in which case the best response curve $R\left(\lambda_{0}\right)$ crosses the 45 -degree line at some $\lambda_{0} \leq \bar{\lambda}_{0}$ ), whereas a mixed-strategy equilibrium exists when this inequality is reversed (in which case the 45-degree line is "crossed" as the best response correspondence jumps at $\left.\bar{\lambda}_{0}\right)$. By definition of $\bar{\lambda}_{0}$ and $\bar{z}$, the sufficient condition for a pure-strategy equilibrium is

$$
R\left(\bar{\lambda}_{0}\right)=\frac{x}{\bar{z}} \leq \bar{\lambda}_{0}=\frac{1}{q} \Phi(\bar{z})
$$

which is the same as

$$
q \leq \bar{q} \equiv \frac{\bar{z} \Phi(\bar{z})}{x}
$$


This condition is not only sufficient but also necessary for a pure-strategy equilibrium provided that $R^{\prime}(\lambda)<1$ holds at any $\lambda=R(\lambda)$ (so that the 45 -degree line can be crossed at most once). This condition then also implies that the Nash equilibrium is unique. As in the proof of Proposition $1, R^{\prime}(\lambda)<1$ holds at any $\lambda=R(\lambda)$ if condition (20) holds. The denominator on the left-hand side is positive because of the second-order condition $\lambda z>1-a$. Hence, the inequality is

$$
-(2-\lambda z)\left[1-e^{-\lambda q}(1+\lambda q)\right]<\lambda q\left[\lambda z^{2}+(a-1) z\right] .
$$

Substitution of $z=\left(1-e^{-\lambda q}\right) /(\lambda q)$ yields

$$
2 \lambda q<\lambda\left(1-e^{-\lambda q}\right)+(1+a)\left(e^{\lambda q}-1\right) .
$$

Again because of the second-order condition $\lambda \geq(1-a) / z=(1-a) \lambda q /\left(1-e^{-\lambda q}\right)$, a sufficient condition for this inequality is that

$$
2 \lambda q<(1-a) \lambda q+(1+a)\left(e^{\lambda q}-1\right) .
$$

This is the same as

$$
0<\left(e^{\lambda q}-1-\lambda q\right)(1+a),
$$

which is obviously true for any $\lambda>0$.

\section{Proof of Proposition 4:}

As in section 7.2 of Rogerson, Shimer, and Wright (2005), it is straigtforward to show that $W($.$) is affine-linear; it takes the form W(u)=w_{0}-S \cdot u$ where $S$ is marginal social value of an employed worker. Differentiation of (15) with respect to $u$ together with the envelope theorem yields

$$
S=p-\tilde{b}+k(\lambda)+c / q+\beta S\left(e^{-\lambda z}-\delta\right) .
$$

The first-order conditions for $\lambda$ and $q$ are

$$
\begin{gathered}
k^{\prime}(\lambda)=\beta e^{-\lambda z} e^{-\lambda q} S, \\
c=\beta e^{-\lambda z}\left[1-(1+\lambda q) e^{-\lambda q}\right] S .
\end{gathered}
$$

These conditions confirm that optimal $\lambda$ and $q$ do not depend on the state variable $u$, so $W($.$) is indeed affine-linear. Both first-order conditions are the same as in$ Proposition 4. The value $S$ follows after substitution of (24) into (22) and solving for $S$. 


\section{References}

Acemoglu, D. (2001): "Good Jobs versus Bad Jobs," Journal of Labor Economics, $19,1-22$.

Albrecht, J., P. Gautier, and S. Vroman (2006): "Equilibrium Directed Search with Multiple Applications," Review of Economic Studies, 73, 869-891.

Albrecht, J., And S. Vroman (2002): "A Matching Model with Endogenous Skill Requirements," International Economic Review, 43, 283-305.

Burdett, K., And Judd (1983): "Equilibrium Price Dispersion," Econometrica, 51, 955-969.

Burdett, K., S. Shi, And R. Wright (2001): "Pricing and Matching with Frictions," Journal of Political Economy, 109, 1060-1085.

Butters, G. (1977): "Equilibrium Distribution of Sales and Advertising Prices," Review of Economic Studies, 44, 465-491.

Den HaAn, W., G. Ramey, and J. Watson (2000): "Job Destruction and Propagation of Shocks," American Economic Review, 90, 482-498.

Galenianos, M., And P. Kircher (2008): "Directed Search with Multiple Job Applications," forthcoming in Journal of Economic Theory.

Gautier, P., and R. Wolthoff (2007): "Simultaneous Search with Heterogeneous Firms and Ex Post Competition," CEPR Discussion Paper 6169.

Hagedorn, M., And I. Manovskit (2007): "The Cyclical Behavior of Equilibrium Unemployment and Vacancies Revisited," forthcoming in American Economic Review.

HALl, R. (1977): "An Aspect of the Economic Role of Unemployment," in $M i$ croeconomic Foundations of Macroeconomics, ed. by G. Harcourt. Macmillan, London.

Julien, B., J. Kennes, And I. King (2000): "Bidding for Labor," Review of Economic Dynamics, 3, 619-649. 
- (2006): "Residual Wage Disparity and Coordination Unemployment," International Economic Review, 47, 961-989.

KIRCHER, P. (2007): "Efficiency of Simultaneous Search," Working Paper, University of Pennsylvania.

Krueger, A., And A. Mueller (2008): "Job Search and Unemployment Insurance: New Evidence from Time Use Data," IZA Discussion Paper No. 3667.

LAgos, R. (2000): "An Alternative Approach to Search Frictions," Journal of Political Economy, 108, 851-873.

Lester, B. (2008): "Directed Search with Multiple Vacancies," Working Paper, University of Western Ontario.

Montgomery, J. (1991): "Equilibrium Wage Dispersion and Interindustry Wage Differentials," Quarterly Journal of Economics, 106, 163-179.

Mortensen, D. (2007): "Island Matching," NBER Working Paper 13287.

Nickell, S., L. Nunziata, and W. Ochel (2005): "Unemployment in the OECD since the 1960s: What Do We Know?," The Economic Journal, 115, 1-27.

Petrongolo, B., and C. Pissarides (2001): "Looking into the Black Box: A Survey of the Matching Function," Journal of Economic Literature, 39, 390-431.

Pissarides, C. (2000): Equilibrium Unemployment Theory. The MIT Press, Cambridge, MA, 2 edn.

Rogerson, R., R. Shimer, And R. Wright (2005): "Search-Theoretic Models of the Labor Market: a Survey," Journal of Economic Literature, 43, 958-988.

ShImer, R. (2004): "Search Intensity," Working Paper, University of Chicago.

- (2005): "The Cyclical Behavior of Equilibrium Unemployment and Vacancies," American Economic Review, 95, 25-49.

(2007): "Mismatch," American Economic Review, 97, 1074-1101. 